\title{
Investigação matemática com o GeoGebra: um exemplo com matrizes e determinantes
}

\section{Duelci Aparecido de Freitas Vaz}

Instituto Federal de Educação, Ciência e Tecnologia de Goiás (IFG)

Pontifícia Universidade Católica de Goiás (PUC GOIÁS)

duelci.vaz@ig.com.br

\section{Elivanete Alves de Jesus}

Pontifícia Universidade Católica de Goiás (PUC GOIÁS)

elivanetea@gmail.com

\section{Resumo}

Nesta sugestão de aula apresentamos uma proposta de investigação matemática sobre matrizes e determinantes, articulada com o software GeoGebra. A ideia, trabalhada com alunos do segundo ano do ensino médio em um laboratório de informática, tem como objetivo mostrar o caráter dedutivo do pensamento matemático na tentativa de generalizar uma propriedade dos determinantes.

Palavras-chave: Investigação Matemática. Ensino Médio. Matrizes. Determinantes. GeoGebra.

\section{Mathematical investigation with GeoGebra: an example with matrices and determinants}

\begin{abstract}
In this class suggestion, we propose a math investigation on matrices and determinants, using GeoGebra software. The idea, apply in a computer lab with sophomore high school students, has as its main goal to show the deductive character of the math thought process, in an attempt to generalize one property of the determinants.
\end{abstract}

Keywords: Mathematic Inquiry. High School. Matrices. Determinants. GeoGebra. 


\section{Introdução}

Com o software GeoGebra podemos contemplar geometria dinâmica e álgebra, interagindo entre si na mesma tela, possibilitando o usuário relacionar as várias faces de um mesmo objeto matemático. O programa permite trabalhar conceitos matemáticos do ensino fundamental, médio e também conceitos de cálculo diferencial, geometria euclidiana, geometria analítica e alterá-los dinamicamente após a construção ser finalizada. Esse dinamismo permite que o aluno perceba diversas relações entre os objetos matemáticos, faça conjecturas e até mesmo formalize os resultados, de forma visual, no próprio software. Além das operações usuais o GeoGebra deriva, integra funções, oferece comandos diversos, entre eles, comandos para encontrar raízes e pontos extremos de uma função. Seu uso tem sido extensamente explorado por diversos professores.

\section{A proposta}

O GeoGebra possibilita um trabalho investigativo para a realização de provas de conjecturas levantadas tanto pelo professor como por alunos. Relatamos aqui uma experiência para ilustrar tal situação. Durante uma aula de matemática, no desenvolvimento de uma atividade, uma estudante nos apresentou uma conjectura, resultado de sua a experiência ao calcular soma de matrizes e determinantes. Nessa atividade, embora sem a utilização do gráfico que o software permite, foi possível, depois de certas experimentações, conjecturar uma propriedade interessante do cálculo de determinantes.

A aluna apresentou-nos alguns exemplos de cálculos de determinantes associados a uma matriz $2 \times 2$. Depois de vários exemplos dados por ela, solicitamos exemplos com matrizes $3 \times 3$. O exemplo imediatamente apresentado foi o de uma matriz com os seguintes elementos consecutivos: $1,2,3,4,5,6,7,8$ e 9, que no GeoGebra, na entrada de comandos, é escrita $\mathrm{M}=\{\{1,2,3\},\{4,5,6\},\{7,8,9\}\}$ e na janela algébrica é representada como nos livros didáticos. Para calcular o determinante, basta escrever na entrada de comandos Determinante [M], dar enter, e obtém-se na janela algébrica seu valor, neste caso zero. A imagem abaixo ilustra essas ideias. 

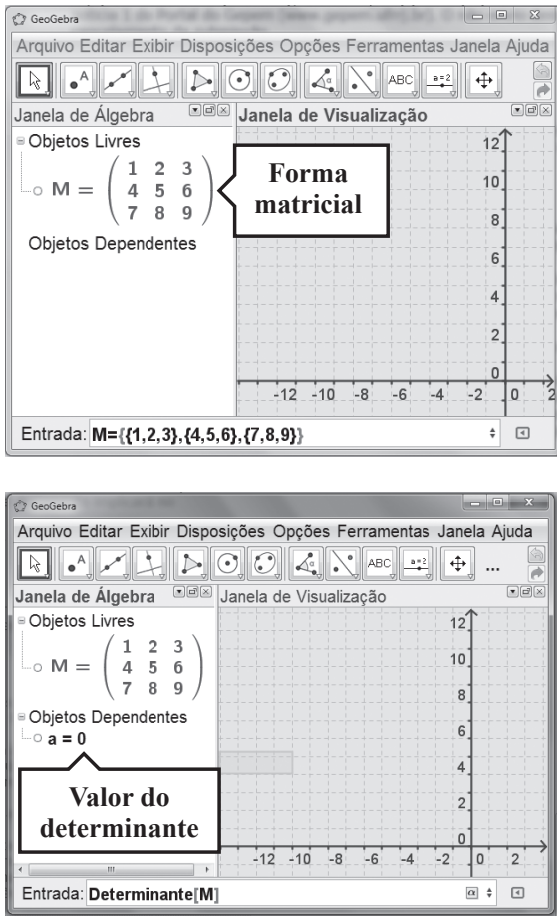

Solicitamos à discente que apresentasse outro caso e foi dado o exemplo de uma matriz com os elementos: 3, 4, 5, 6, 7, 8, 9, 10 e 11, com determinante também igual a zero. Após a realização de vários cálculos com números consecutivos, os alunos conjecturaram algo interessante: o determinante de matrizes com elementos consecutivos é sempre zero.

Então pedimos aos estudantes para verificarem se isso era um fato geral. Solicitamos uma prova da conjectura. Muitos alunos voltaram a experimentar no GeoGebra diversos outros exemplos com a característica citada, constatando que o valor desses determinantes eram sempre zero. Também foram incorporados sequências de números decimais, como por exemplo, $\mathrm{M}=\{\{2.5,3.5,4.5\},\{5.5,6.5,7.5\}$, $\{8.5,9.5,10.5\}\}$, confirmando a conjectura inicial. Ampliando nossa investigação, sugerimos que escrevessem na entrada de comando $M=\{\{n, n+1, n+2\},\{n+3, n+4$, $\mathrm{n}+5\},\{\mathrm{n}+6, \mathrm{n}+7, \mathrm{n}+8\}\}$, fazendo o valor de $\mathrm{n}$ variar no intervalo [-5,5], também confirmando a conjectura. Isso, entretanto, não pode ser usado como a demonstração formal do fato, pois neste caso, embora a variável assuma diversos valores dentro de um intervalo, não assume todos esses valores. Além do mais, os valores externos ao intervalo ficaram excluídos da verificação. 


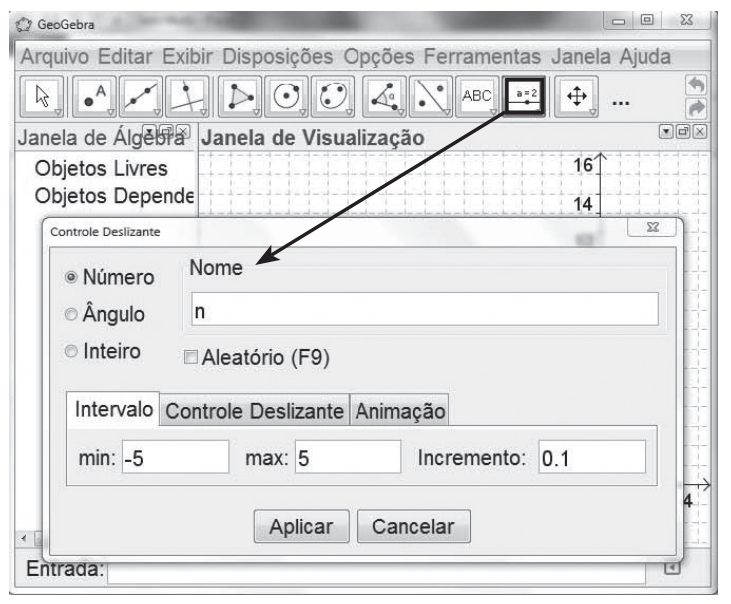

Selecione controle deslizante, clique na janela gráfica, defina $n$ variando de -5 a 5 .

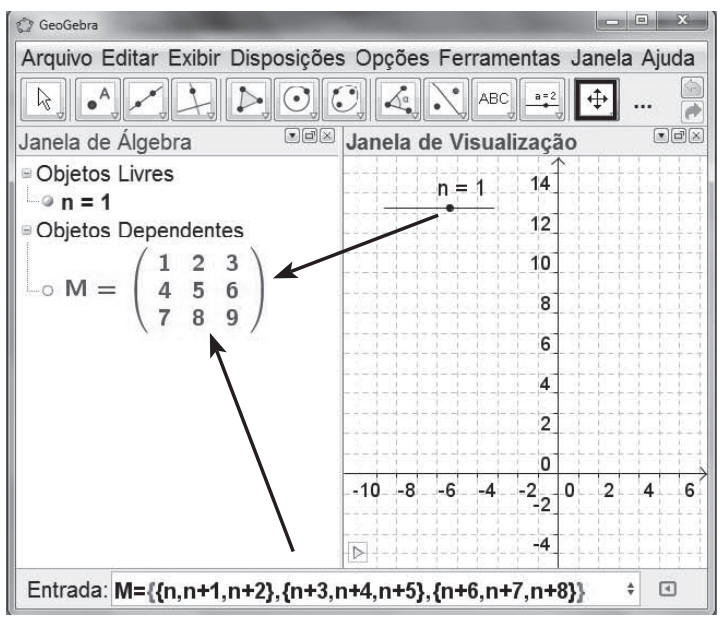

Com o lado direito do mouse sobre $\mathbf{n}$, anime-o. Os valores da matriz $M$ correspondem ao valor $n=1$.

Ao continuar investigando o caso, exploramos o fato de que toda propriedade válida para linhas também é válida para colunas. A partir de então passamos a trabalhar com números consecutivos em forma de colunas.

Para demonstrar a validade da conjectura, para o caso $3 \times 3$, procedemos aplicando propriedades dos determinantes e percebemos que a primeira proposta, para confirmar a conjectura, não foi bem assimilada pelos alunos, embora fosse adequada à série trabalhada. Assim, aproveitamos o momento para explorá-la e comprovar a validade da conjectura. Na realização dessa tarefa foi percebido por todos que a variável $n$ pode ser um número real qualquer: 


$$
\begin{aligned}
\left|\begin{array}{ccc}
n & n+3 & n+6 \\
n+1 & n+4 & n+7 \\
n+2 & n+5 & n+8
\end{array}\right|=\left|\begin{array}{ccc}
n & n & n+6 \\
n+1 & n+1 & n+7 \\
n+2 & n+2 & n+8
\end{array}\right|+\left|\begin{array}{ccc}
n & 3 & n+6 \\
n+1 & 3 & n+7 \\
n+2 & 3 & n+8
\end{array}\right| \\
=\left|\begin{array}{ccc}
n & 3 & n+6 \\
n+1 & 3 & n+7 \\
n+2 & 3 & n+8
\end{array}\right|=\left|\begin{array}{ccc}
n & 3 & 6 \\
n+1 & 3 & 6 \\
n+2 & 3
\end{array}\right|+\left|\begin{array}{ccc}
n & 3 & n \\
n+1 & 3 & n+1 \\
n+2 & 3 & n+2
\end{array}\right|=0
\end{aligned}
$$

Também aproveitamos o momento para explorar outras propriedades dos determinantes, mostrando que o cálculo poderia ser efetuado por outras vias. $\mathrm{Na}$ realização do raciocínio abaixo ficou evidenciado um entendimento melhor da propriedade:

$$
\left|\begin{array}{ccc}
n & n+3 & n+6 \\
n+1 & n+4 & n+7 \\
n+2 & n+5 & n+8
\end{array}\right|=\left|\begin{array}{ccc}
n & n+3 & n+6 \\
1 & 1 & 1 \\
2 & 2 & 2
\end{array}\right|=0
$$

Num cenarıo ue mvesugaçao, a pergunıa sequenciaı serıa sodre a validade dessa regra para matrizes de outra ordem. Incentivamos primeiramente o caso $4 x 4$. Os alunos fizeram diversas experimentações no GeoGebra, como anteriormente, comprovando que a mesma conjectura era válida neste caso também. A formalização foi realizada como mostra o cálculo a seguir. Aqui a propriedade também foi explorada em mais de uma via, em consórcio com propriedades dos determinantes, articulando com conhecimentos desenvolvidos anteriormente. A primeira demonstração do fato foi feita como segue:

$$
\begin{aligned}
\left|\begin{array}{cccc}
n & n+4 & n+8 & n+12 \\
n+1 & n+5 & n+9 & n+13 \\
n+2 & n+6 & n+10 & n+14 \\
n+3 & n+7 & n+11 & n+15
\end{array}\right|= \\
=\left|\begin{array}{cccc}
n & n & n+8 & n+12 \\
n+1 & n+1 & n+9 & n+13 \\
n+2 & n+2 & n+10 & n+14 \\
n+3 & n+3 & n+11 & n+15
\end{array}\right|+\left|\begin{array}{cccc}
n & 4 & n+8 & n+12 \\
n+1 & 4 & n+9 & n+13 \\
n+2 & 4 & n+10 & n+14 \\
n+3 & 4 & n+11 & n+15
\end{array}\right|
\end{aligned}
$$

$$
\begin{aligned}
=\left|\begin{array}{cccc}
n & 4 & 4 & \mathrm{n}+12 \\
\mathrm{n}+1 & 4 & 4 & \mathrm{n}+13 \\
\mathrm{n}+2 & 4 & 4 & \mathrm{n}+14 \\
\mathrm{n}+3 & 4 & 4 & \mathrm{n}+15
\end{array}\right|+\left|\begin{array}{cccc}
\mathrm{n} & 4 & \mathrm{n}+4 & \mathrm{n}+12 \\
\mathrm{n}+1 & 4 & \mathrm{n}+5 & \mathrm{n}+13 \\
\mathrm{n}+2 & 4 & \mathrm{n}+6 & \mathrm{n}+14 \\
\mathrm{n}+3 & 4 & \mathrm{n}+7 & \mathrm{n}+15
\end{array}\right| \\
=\left|\begin{array}{ccccc}
\mathrm{n} & 4 & \mathrm{n} & \mathrm{n}+12 \\
\mathrm{n}+1 & 4 & \mathrm{n}+1 & \mathrm{n}+13 \\
\mathrm{n}+2 & 4 & \mathrm{n}+2 & \mathrm{n}+14 \\
\mathrm{n}+3 & 4 & \mathrm{n}+3 & \mathrm{n}+15
\end{array}\right|+\left|\begin{array}{cccc}
\mathrm{n} & 4 & 4 & \mathrm{n}+12 \\
\mathrm{n}+1 & 4 & 4 & \mathrm{n}+13 \\
\mathrm{n}+2 & 4 & 4 & \mathrm{n}+14 \\
n+3 & 4 & 4 & \mathrm{n}+15
\end{array}\right|=0
\end{aligned}
$$


A outra forma escolhida para demonstrar o fato está ilustrada abaixo:

$$
\left|\begin{array}{cccc}
n & n+4 & n+8 & n+12 \\
n+1 & n+5 & n+9 & n+13 \\
n+2 & n+6 & n+10 & n+14 \\
n+3 & n+7 & n+11 & n+15
\end{array}\right|=\left|\begin{array}{cccc}
n & n & n+8 & n+12 \\
1 & 1 & 1 & 1 \\
2 & 2 & 2 & 2 \\
3 & 3 & 3 & 3
\end{array}\right|=0 .
$$

Para uma matriz quadrada de ordem k qualquer, maior ou igual que 3, podemos demonstrar como segue:

$$
\begin{gathered}
\left|\begin{array}{ccccc}
n & n+k & n+2 k & \ldots & n+(k-1) k \\
n+1 & n+k+1 & n+2 k+1 & \ldots & n+(k-1) k+1 \\
\ldots & \ldots & \ldots & \ldots & \ldots \\
n+k-1 & n+2 k-1 & n+3 k-1 & \ldots & n+k^{2}-1
\end{array}\right|= \\
\left|\begin{array}{ccccc}
n & n+k & n+2 k & \ldots & n+(k-1) k \\
1 & 1 & 1 & \ldots & 1 \\
\ldots & \ldots & \ldots & \ldots & \ldots \\
k-1 & k-1 & k-1 & \ldots & k-1
\end{array}\right|=0 .
\end{gathered}
$$

A dificuldade neste caso ficou relacionada a ordenar os termos. Mas tal dificuldade foi superada observando casos particulares.

Restava-nos investigar as matrizes $2 \times 2:\left|\begin{array}{cc}n & n+2 \\ n+1 & n+3\end{array}\right|=n^{2}+3 n-n^{2}-$, $2 n-n-2=-2$, constatando que, para esse caso, o determinante é sempre igual a -2 .

Assim, foi possível enunciar a regra geral. Toda matriz quadrada de ordem maior ou igual a 3, em que os elementos estão dispostos consecutivamente, em forma de linhas ou de colunas, possui determinante nulo. No caso especial em que a matriz tem ordem 2 , o determinante é sempre -2 .

\section{Referência}

GeoGebra2012. < http://www.geogebra.org/cms/pt_BR/download >. Acesso: 12/02/2012. 